\title{
Utilização de Morfismo como Classificador para Verificação de Assinaturas Off-line
}

\author{
Lucindo de Mélo, Cleber Zanchettin, Anderson Lira Queiroz, Gliner Dias Alencar
}

Centro de Informática, Universidade Federal de Pernambuco

Recife, PE 50.732-970, Brazil

lucindomelo@gmail.com, \{cz, aalq, gda2\}@cin.ufpe.br

\begin{abstract}
This paper provides contributions to the problem of Off-line signature verification by creating a classifier based on morphism using a technique of triangulation of points. This technique allows checking the distance between the signatures of a particular class, aiming to discrimine false from genuine signatures. In this paper, five scenarios were evaluated using genuine, simulated and random fakes signatures. The fifth scenario use a variable amount of points per experiment, which one obtained the best result, reaching a Global error rate of 0.18 .

Resumo. Esta artigo oferece contribuições para o problema de verificação de assinaturas Off-line através da criação de um classificador baseado em morfismo utilizando uma técnica de triangulação de pontos. Esta técnica permite verificar a distância entre as assinaturas de uma determinada classe, objetivando discriminá-las como falsas ou genuínas. Neste artigo cinco cenários foram avaliados, em todos foram utilizadas assinaturas genuínas, falsificações simuladas e falsificações aleatórias. $O$ quinto cenário é avaliado utilizando uma quantidade variável de pontos por experimento, no qual foi obtido o melhor resultado, uma taxa de Erro Global de 0,18.
\end{abstract}

\section{Introdução}

A verificação de assinatura é uma tarefa necessária na sociedade, assinaturas são bem estabelecidas e aceitas como um meio formal de verificação da identidade de pessoas. Devido a essa característica, elas são usadas por governos, transações comerciais e legais, sendo um dos métodos mais aceitos de verificação da identidade [BARANOSKI, et. al., 2005].

A verificação de assinaturas reduz o risco de uma assinatura forjada ser aceita como verdadeira. O problema que surge quando a validação da assinatura muitas vezes precisa ser feita rapidamente. Isso geralmente ocorre quando se lida com transações monetárias, especialmente através de cartões de crédito e cheques bancários [MA, 2007]. Uma vantagem da utilização de assinaturas é a segurança, pois ao contrário das senhas, mesmo que alguém conheça a assinatura de um usuário, usualmente não é possível reproduzir esta assinatura de forma trivial.

A assinatura manuscrita, assim como a escrita manuscrita, é um comportamento biométrico, o qual sofre variação em todo período de tempo na vida de um indivíduo. Características físicas e psicológicas influenciam vigorosamente na formação de tais comportamentos, além disso, a assinatura de um indivíduo é socialmente e legalmente aceita como um firmamento de intenção ou anuência sobre algo [BERTOLINI, 2008]. 
Apesar das diversas vantagens, a autenticação automática de assinaturas é um problema de difícil solução do ponto de vista computacional, devido à grande variabilidade existente entre assinaturas de uma mesma pessoa. Este foi um dos fatos que inspirou pesquisadores a estudar duas categorias distintas de sistemas de verificação automática de assinatura. Estes sistemas são referidos como Off-line e On-line [BALTZAKIS \& PAPAMARKOS, 2000], [YEUNG, et. al., 2004]. No primeiro caso a assinatura é avaliada posteriormente a sua escrita e no segundo caso no momento da escrita, a qual é adquirida em um dispositivo específico (ex. tablet).

No contexto do reconhecimento de assinaturas Off-line, a abordagem proposta neste artigo utiliza uma técnica de computação gráfica conhecida como Morphing, para desenvolver uma metodologia e um classificador capaz de identificar os diversos tipos de falsificações existentes.

\section{Verificação de Assinaturas Manuscristas}

Um sistema de verificação de assinaturas objetiva autenticar uma assinatura através de métodos que possam discriminar amostras genuínas de falsificações [BERTOLINI, 2008]. De acordo com o método de aquisição da mesma, o processo de verificação de assinaturas pode ser classificado como On-line ou Off-line. A diferença entre os métodos On-line (Dinâmica) e Off-line (Estática) se caracteriza pela forma como são obtidas as assinaturas [PLAMONDON \& LORETTE, 1989].

\subsection{Tipos de Falsificações}

De acordo com Coetzer [COETZER, 2005], pode-se classificar as falsificações de assinaturas em três tipos: aleatória, simples e simulada. Observa-se um aumento da qualidade nas falsificações quando se desloca das aleatórias em sentido das simuladas, dificultando seu reconhecimento, conforme apresentado na Figura 1.

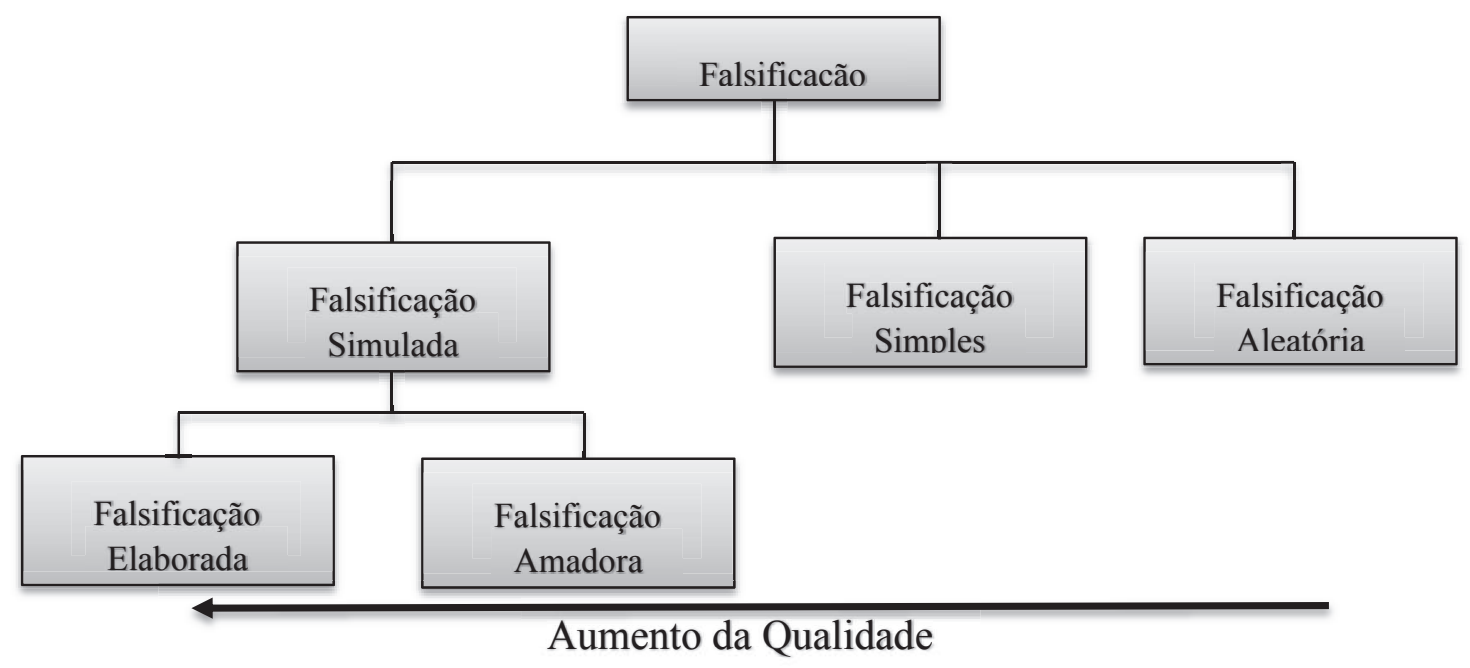

Figura 1: Tipos de Falsificação. Adaptação de [COETZER, 2005].

\subsection{Medidas de Desempenho}

O desempenho dos sistemas de verificação de assinaturas pode ser avaliado em função dos erros ocorrido quanto à classificação. Na Figura 2, são apresentadas duas distribuições normais, sendo classes genuínas e falsificações. Observa-se uma 
intersecção entre essas curvas. A área preenchida da intersecção representa os erros do sistema.

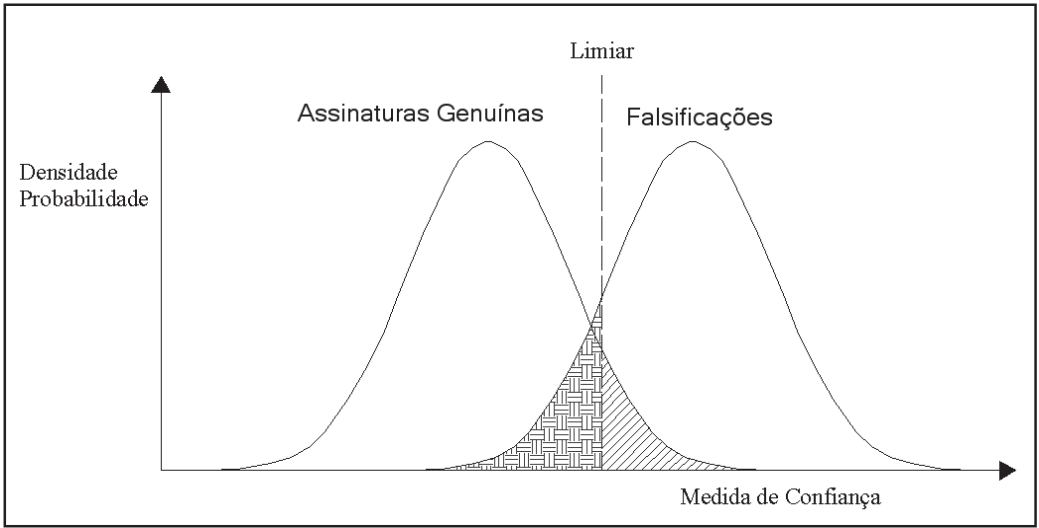

Figura 2: Intersecção existente entre assinaturas genuínas e falsificações [Bertolini, 2008].

Na taxa de Falsa Rejeição (False Rejection Rate - FRR) ou Falso Negativo ou erro tipo I, o sistema classifica uma assinatura como falsa, sendo ela uma assinatura genuína.

$$
\mathbf{F R R}=\frac{\text { Número de Assinaturas Genuínas Rejeitadas }}{\text { Número de Assinaturas Genuínas Submetidas }}
$$

Na taxa de Falsa Aceitação (False Acceptance Rate - FAR) ou Falso Positivo ou erro tipo II, o sistema classifica, de forma verdadeira, sendo ela uma assinatura não genuína.

$$
\mathbf{F A R}=\frac{\text { Número de Falsificações Aceitas }}{\text { Número de Falsificações Submetidas }}
$$

Alguns autores como (Coetzer, 2005), apresentam ainda a Taxa de Erro Médio (Average Error Rate - AER), que representa a média aritmética entre FRR e FAR.

$$
\mathbf{A E R}=\frac{\mathrm{FRR}+\mathrm{FAR}}{2}
$$

\subsection{Sistemas de Reconhecimento de Padrões}

Um classificador para verificação de assinaturas é um sistema que através de um vetor de características que representam a assinatura é capaz de identificar se uma nova assinatura que lhe é apresentada pertence ou não ao indivíduo que se supõe que ela pertença. Essa resposta do sistema pode ser binária, indicando apenas se a assinatura é ou não verdadeira, ou pode ainda indicar um percentual, ou um grau de veracidade [SOUSA, 2009].

\section{Método para utilização de morfismo como classificador para verificação de assinaturas off-line}

Na Figura 3 é apresentado um resumo do processo de verificação de assinaturas proposto.

\subsubsection{Segmentação}

$\mathrm{Na}$ proposta deste artigo a segmentação tem grande relevância, pois o classificador desenvolvido pode utilizar diferentes tamanhos de grades para realizar a verificação da assinatura. A configuração do problema proposto adéqua-se melhor na abordagem não 
contextual para a segmentação das assinaturas. Essa abordagem faz uso de características relacionadas à forma dos traços das assinaturas, levando em consideração os aspectos geométricos e estatísticos desses traços, se tornando mais adequada para sistemas de verificação de assinaturas, devido à grande quantidade de rubricas, estilos diferentes utilizados, pois permite a utilização de diferentes técnicas.

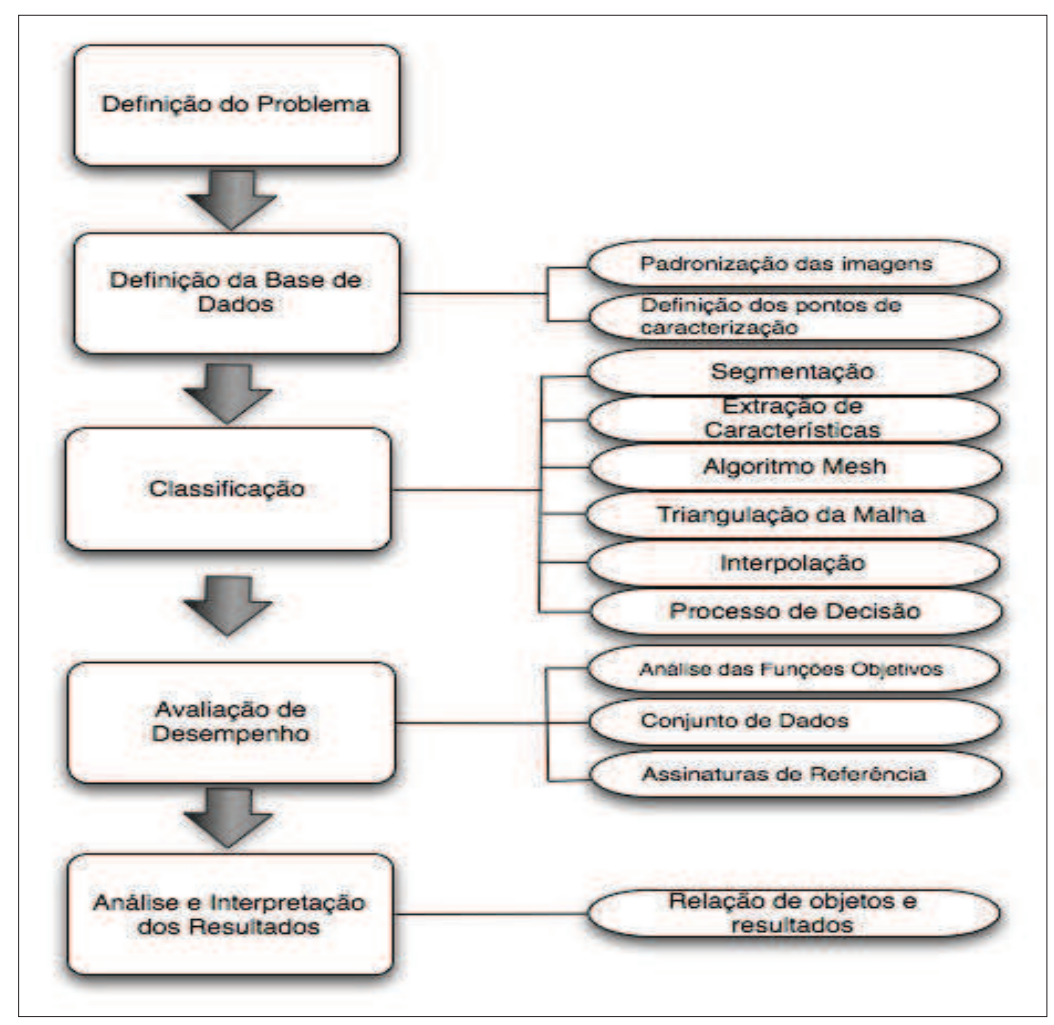

Figura 3: Método de Classificação por Morfismo.

As grades (grids) utilizadas neste artigo têm a características de se moldarem ao formato da imagem, funcionando como uma impressão digital para cada classe imagem, um exemplo pode ser observado na Figura 4.

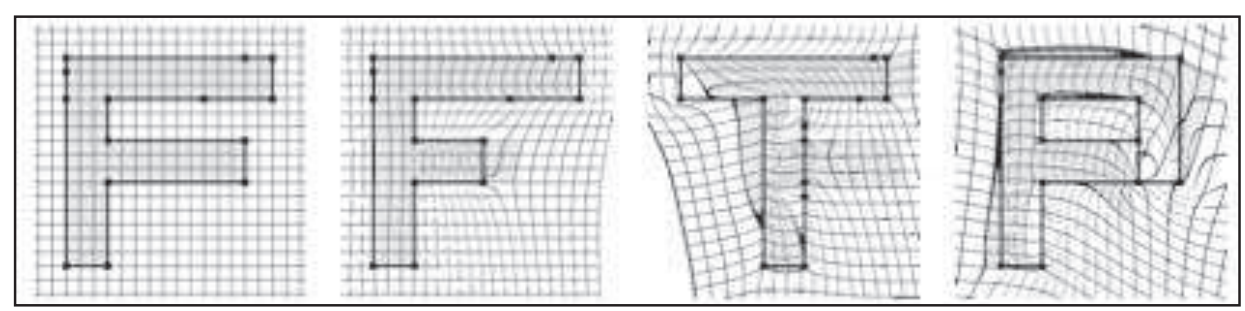

Figura 4: Configuração do grid para cada tipo de imagem, modelo proposto.

Após o treinamento ser realizado, cada classe de assinatura passa a ter uma formatação de grid específica, característico apenas dessa classe. Na Figura 4 pode-se observar a distorção gerada na grid para transformar a imagem $\mathrm{F}$ original em outra imagem F, como também se podem ver as configurações finais das grids, geradas a partir de $\mathrm{F}$ e transformada em T e P. Conclui-se então que imagens semelhantes geram uma perturbação menor na grid, enquanto que imagens de classes diferentes a distorção é maior. 


\subsubsection{Extração de Características}

Kass e Terzopoulos [KASS, WITKIN \& TERZOPOULOS, 1988], descreveram um método de contornos ativos, que chamaram modelo de snake. Contornos ativos são modelos geométricos em que suas coordenadas são os parâmetros do modelo. O snake tornou-se uma ferramenta inestimável para base de segmentação e rastreamento de objetos, capaz de estabilizar a sua evolução na proximidade de bordas, linhas ou terminações em imagens. Observa-se na Figura 5 os pontos criados pelo snake.

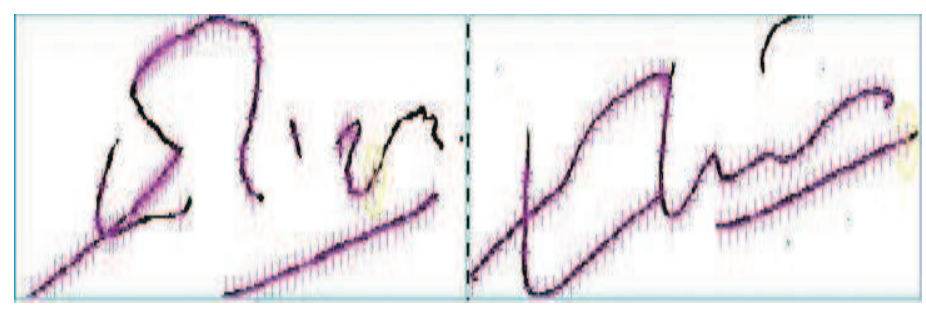

Figura 5: Pontos de controle gerados pelo snake.

\subsubsection{Algoritmo Mesh entre as Etapas do Morfismo}

Após a obtenção dos pontos (snake), a etapa seguinte é a realização do morfismo (morph) entre duas imagens. Tem-se assim, a assinatura da esquerda (left), na qual se deseja fazer a transformação, e a assinatura direita (right), o resultado da transformação. A partir da interseção entre a grid e o snake de cada imagem obtém-se uma malha (mesh) onde os pontos estão localizados nos vértices do grid, então é feito uma correlação de um-para-um (1:1) entre os pontos das duas assinaturas. Realizando-se a fusão entre as duas malhas (mesh merging) obtidas, como pode ser visto na Figura 6.

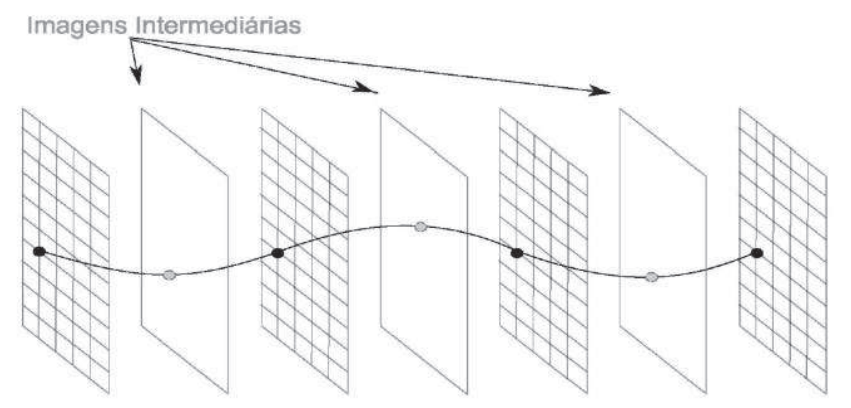

Figura 6: Etapas no morfismo

\subsubsection{Triangulação dos Retângulos da Malha}

A Triangulação de Delaunay [DELAUNAY, 1934] e [WEISSTEIN, 2011] tem a seguinte propriedade: ele determina um círculo cujo interior não contém nenhum outro ponto do conjunto de pontos que não seja os três pontos que determinam o triângulo. Esta condição significa que, dentro do círculo circunscrito de um triângulo, não deve haver ponto que não pertence a este triângulo.

Segundo [GUEDES, 2011] o custo computacional para realizar a triangulação de Delaunay pode chegar, no pior caso a uma complexidade $\mathrm{O}\left(\mathrm{n}^{2}\right)$, porém é considerado por Guedes um bom algoritmo. Ainda em Guedes se um certo problema é dividido exatamente ao meio em um algoritmo, com a técnica dividir-para-conquistar, e os procedimentos para a divisão e unificação juntos têm complexidade $\mathrm{O}(\mathrm{n})$, então o 
algoritmo tem complexidade $\mathrm{O}(\mathrm{n} \log \mathrm{n})$, e se esses procedimentos tem complexidade $\mathrm{O}(1)$, então o algoritmo tem complexidade $\mathrm{O}(\log n)$.

\subsubsection{Interpolação de Triângulo}

A interpolação é utilizada para deformar as coordenadas de cada ponto de um triângulo de entrada para o ponto correspondente de um triângulo resultado. Isso é feito por uma matriz de transformação com duas linhas e três colunas. Para cada ponto da imagem resultado, os pontos correspondentes das imagens de entrada são calculados inversamente com as seguintes fórmulas: xinput $=$ a11 *xresult + a12 $*$ yresult + a13 (3.1); yinput $=a 21 *$ xresult $+a 22 *$ yresult $+a 23$ (3.2).

Todo o triângulo pode ser transformado pela matriz. Pontos, área e linha são interpolados, além da transformação afim como transladar, rotacionar e dimensionar o sistema de coordenadas de um triângulo não ortogonal. Pode-se obter a matriz de uma transformação afim entre um único par de triângulos através da resolução de um sistema de seis equações com seis variáveis, exemplificada na Figura 7.

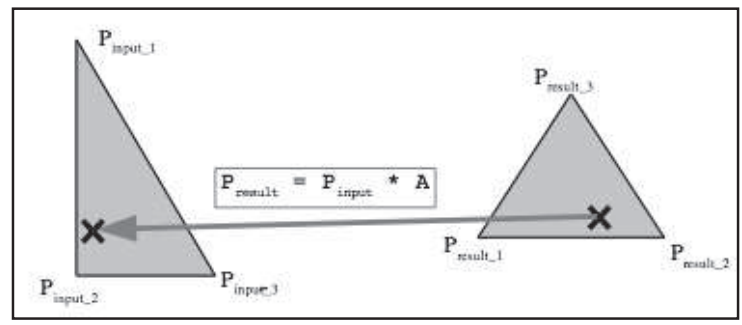

Figura 7: Interpolação de Triangulo

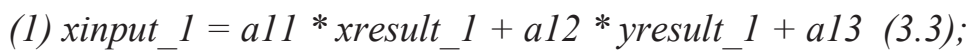

(2) yinput_ $_{-} 1=a 21 *$ xresult_ $1+$ a22 $*$ yresult_ $1+a 23$ (3.4);

(3) xinput $2=a 11 *$ xresult $2+$ a12 $*$ yresult $2+$ al3 (3.5);

(4) yinput_2 $=a 21 * x$ result_ $2+a 22 *$ yresult_ $2+a 23$ (3.6);

(5) $x$ input_3 $=a 11 * x$ result_3 + a12 $*$ yresult_ $3+$ al3 (3.7);

(6) yinput_3 $=a 21 * x$ result_3 + a22 $*$ yresult_3 $+a 23$ (3.8).

Os pontos são transformados e todos os pontos pertencem a um índice do triângulo na lista. Na Figura 8(a) é mostrada a interpolação entre duas assinaturas da mesma classe, a Figura 8(b) mostra a interpolação entre duas assinaturas de classes diferentes. Foram geradas, através de interpolação linear, duas imagens intermediarias.

O processo de transformação de uma assinatura em outra pode ser realizado em várias etapas de interpolação, fazendo com que a transformação fique mais suave, ou seja, são geradas várias imagens de interpolação intermediárias. 


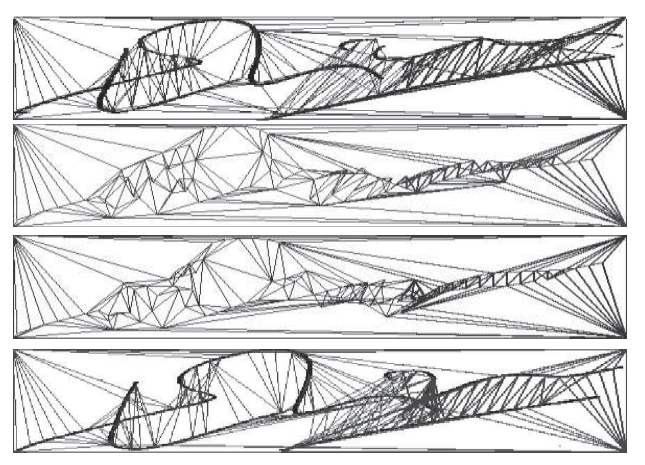

(a)

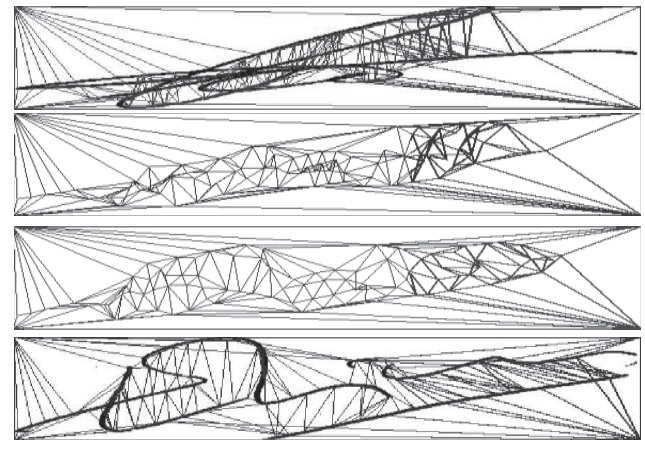

(b)

Figura 8: Interpolação linear entre duas assinaturas

\subsubsection{Complexidade Algorítmica}

O custo computacional para realizar a verificação de assinaturas, utilizando morfismo, é equivalente a soma das complexidades de realização do snake $\mathrm{O}(\mathrm{n})$, triaungulação de Delaunay $\mathrm{O}(\log n)$ e interpolação de triangulo $\mathrm{O}(\mathrm{n})$, logo, tem-se, $\mathrm{O}(\mathrm{n})+\mathrm{O}(\log n)+$ $\mathrm{O}(\mathrm{n})$.

\subsubsection{Distância Euclidiana}

A Distância Euclidiana é derivada do cálculo da distância geométrica entre dois pontos, sendo adequada para o tratamento de classes cujos elementos tendem a se agrupar próximos à média, ou seja, possuem variância desprezível. Problemas em que as classes apresentam comportamento semelhante quanto à forma da função de distribuição de probabilidades e valores de variância também são indicados para o uso da Distância Euclidiana [BARBEAU et al., 2002].

$$
L d_{i j}=\sum \sqrt{\left(\mathrm{X}_{\mathrm{ik}-\mathrm{X}} \mathrm{Xjk}\right)^{2}}
$$

\subsubsection{Processo de Decisão}

Entre as etapas da interpolação das assinaturas é calculada a distância entre os deslocamentos dos vértices dos triângulos da imagem esquerda até a transformação da imagem da assinatura direita, ou seja, parametrizando com um número de $\mathrm{n}$ de saltos a transformação das assinaturas esquerda em direita, teremos um número de n-1 interpolações entre as imagens, tendo um vetor contendo todos os pontos dos vértices da triangulação.

Com isso, permite-se calcular exatamente para cada interpolação qual foi à distância deslocada de um ponto em relação a sua posição imediatamente anterior. Para $\mathrm{n}$ saltos são criados $\mathrm{n}$ vetores de tamanho igual ao numero de pontos interpolados, sendo calculadas as distâncias Euclidianas das diferenças entre os saltos, e armazenadas nestas posições.

O treinamento calcula e armazena, nos n vetores, a distância Euclidiana obtida dos pontos em uma mesma classe de assinaturas. Para a fase de testes é calculada e armazenada, nos $\mathrm{n}$ vetores, a distância da assinatura que se deseja avaliar, com as 
assinaturas pertencentes à classe que se deseja verificar sua validade. O processo de decisão sobre a autenticidade de uma assinatura pode ser realizado usando a abordagem de Limiar de Decisão.

Limiar de Decisão: é obtido utilizando, a média da Distancia Euclidiana de todos os pontos dos $\mathrm{n}$ vetores de treinamento, caso a média da Distância Euclidiana dos $\mathrm{n}$ vetores da assinatura a ser submetida ao teste, seja inferior ao limiar a assinatura será considerada genuína.

\section{Experimentos e resultados}

Nesta seção serão demonstrados os experimentos realizados e os resultados obtidos a partir da metodologia proposta. Iniciamos pela aquisição e apresentação da base de dados, em seguida é explanada como foi realizado o pré-processamento das imagens e como ela foi dividida para os experimentos, como também é informada a escolha dos parâmetros e os resultados obtidos em cada experimento. Finalmente, para cada modelo de experimento são escolhidos os melhores resultados para em seguida compará-los, buscando avaliar os resultados entre todos os experimentos.

As assinaturas foram obtidas dos repositórios da competição da ICDAR 2011 Signature Verification Competition. A coleção contém amostras de assinatura on-line e off-line. Os conjuntos de dados off-line serão constituídos de imagens PNG (Portable Network Graphics é um formato de dados utilizado para imagens), digitalizados em 400 DPI (significa Dots Per Inch, em português Pontos por Polegada,) de cor RGB (sigla do sistema de cores aditivas formado pelas iniciais das cores em inglês Red, Green e Blue, que significa em português, respectivamente, Vermelho, Verde e Azul). O conjunto de assinaturas utilizado, composto apenas por autores Ocidentais, sendo eliminadas as de autores Orientais. O total utilizado de assinaturas foi de 360.

\subsection{Pré-Processamento}

O pré-processamento elaborado, para esse estudo, pode ser dividido nas seguintes etapas: No processo de binarização da imagem em preto e branco, pois o conjunto de dados está em 400 DPI de cor RGB e o redimensionamento das imagens, padronizando todas em tamanho 588x117, pois para realizar o morfismo, faz-se necessário que as imagens de uma mesma classe tenham o mesmo tamanho.

\subsection{Cenários dos Experimentos}

Nos experimentos é avaliado o impacto proporcionado por diferentes números de pontos snake sobre as assinaturas no processo de treinamento e teste. A base de dados foi selecionada em 10, 40, 70 e 100 pontos por assinatura para cada autor no treinamento. Foram usadas nos testes, seis assinaturas genuínas, quatro assinaturas de autores diferentes e quatro assinaturas hábeis para cada autor, totalizando 14 assinaturas.

Cinco cenários foram avaliados, em todos foram utilizadas, assinaturas genuínas, falsificações simuladas e aleatórias. Do primeiro ao quarto cenários examinam-se quanto à quantidade fixa de pontos por experimento. $O$ quinto cenário é avaliado utilizando uma quantidade variável de pontos por experimento, ou seja, foi utilizado o melhor resultado para cada classe de assinaturas obtidos nos experimentos com a quantidade de pontos fixas. 
Analisou-se também o impacto que o número de assinaturas usadas como referência nos experimentos influencia no desempenho do sistema. Primeiro utilizou-se 200 assinaturas, sendo 120 genuínas, 40 falsificações aleatórias, 40 falsificações simuladas, em segundo, 240 assinaturas, sendo 180 genuínas, 30 falsificações aleatórias, 30 falsificações simuladas, e o terceiro usou-se como referência 120 assinaturas, sendo 60 genuínas, 30 falsificações aleatórias, 30 falsificações simuladas. Utilizou-se uma base de assinaturas para teste composta por 10 autores.

\subsection{Experimentos e Análise}

Diferentes quantidades de pontos por assinatura foram utilizadas, em cada autor foram disponibilizados arquivos XML contendo 10, 40, 70 e 100 pontos. Para uma melhor análise dividiu-se os experimentos por quantidade de pontos, buscando identificar o melhores resultados na relação quantidade de pontos e assinatura de autor.

Após as analises das diferentes quantidades de pontos, procurou-se neste artigo realizar testes, utilizando a quantidade de melhor desempenho por assinatura de autor. Isso foi possível porque utilizando Morfismo podemos realizar treinamentos e testes independentes por assinatura, não ficando preso a uma estrutura classificadora que engloba todas as assinaturas, como é o caso de Redes Neurais Artificiais. Nos treinamentos e testes pode-se verificar qual foi à melhor configuração do classificador para um determinado autor e utilizar essa informação para futuros testes.

Para determinar o limiar de classificação foram utilizados a soma, média e Distancia Euclidiana de todos os pontos dos n vetores de treinamento, com o intuito de verificar a que obtinha o melhor resultado. A avaliação do impacto que o número de assinaturas de referências no experimento influi nas taxas, é um dos objetivos desta pesquisa, foram utilizadas nos experimentos as seguintes quantidades como referências As $=\{6,12$, e 18$\}$, respectivamente. Através do uso de diferentes números de assinaturas como referência, fica possível estipular até que ponto a quantidade de assinaturas influencia na taxa de acertos, já que na prática o número de assinaturas é um tanto quanto limitado.

Avaliando os resultados finais proporcionados através dos diferentes esquemas, foi observado que o Limiar de Decisão com o uso de Distância Euclidiana apresentou um desempenho superior às demais, o mesmo não ocorrendo para soma e média.

Em cada cenário três casos foram avaliados isoladamente. No primeiro, utilizaram-se seis assinaturas como referência $(\mathrm{As}=6)$, no segundo, 12 assinaturas $(\mathrm{As}=12)$ e por fim 18 assinaturas $(A s=18)$. O classificador foi configurado com uma divisão de grids de 12 X 15 e com geração de duas imagens intermediarias no Morfismo. Essa configuração foi utilizada para todas as análises realizadas neste artigo.

Os resultados alcançados com o Limiar de Decisão para Soma e Média, não foram satisfarório, pois a Taxa de Erro Global em ambos foi de 0,5. Não sendo significativo para análise desta pesquisa. Os experimentos realizados com utilização de Distância Euclidiana associado ao uso do Limiar de Decisão, podem ser vistos, na Tabela 1, foram realizados nos experimentos utilizando 10, 40, 70 e 100 pontos por assinatura.

Ao analisar os resultados da Tabela 1 pode-se concluir que utilizando uma quantidade de pontos específica para cada autor, a quantidade melhor que representa 
uma classe de assinatura, obtem-se um FAR com $25 \%$ e FRR com 11\%, chegando a um AER com 18\%, desta forma melhorarando a confiabilidade em relação ao desempenho do Morfismo como classificador em verificação de assinatura Off-line.

Tabela 1: FAR: False Acceptance Rate; FRR: False Rejection Rate; AER: Average Error Rate.

\begin{tabular}{|c|c|c|c|c|c|}
\hline & $\mathbf{1 0}$ Pontos & 40 Pontos & $\mathbf{7 0}$ Pontos & 100 Pontos & Pontos por Autor \\
\hline FAR & 0,4500 & 0,3667 & 0,3667 & 0,2834 & 0,2500 \\
\hline FRR & 0,1000 & 0,2500 & 0,2833 & 0,2667 & 0,1167 \\
\hline EAR & 0,2750 & 0,3083 & 0,3250 & 0,2750 & 0,1833 \\
\hline
\end{tabular}

\section{Trabalhos Relacionados}

Justino em [JUSTINO, et. al., 2005], utilizou grids fixas sobrepostos à imagem para processo de segmentação, assim, características estáticas e pseudo-dinâmicas são utilizadas. O autor utilizou a Densidade de Pixels, Distribuição de Pixels, Curvatura dos Ângulos e a Inclinação como características estáticas e pseudo-dinâmicas. Diferente da nossa abordagem, onde utilizamos grids que são moudáveis a transformação (morfismos) de uma imagem em outra, ver figura 8.

SHUKLA em [SHUKLA et al. 2014] utiliza o termo "centro de gravidade", que é usado num campo de gravidade uniforme para representar o ponto único em um objeto ou sistema. A noção de centro de massa é a média das massas divididas por suas distâncias de um ponto de referência. Para tanto ele faz uso de Distância Euclidiana para encontrar tais pontos. Neste estudo, Shukla também utilizou triangulação Delaunay nas grids, para encontrar o custo do perímetro de cada triângulo gerado. No presente trabalho, o uso da Distância Euclidiana é utilizada como limiar de decisão, enquanto Triangulação de Delaunay é utilizada na criação da grid.

\section{Conclusão}

Após todos os experimentos realizados, com utilização de diversos pontos por assinatura para uso do classificador proposto, foram avaliados os resultados quanto aos cenários, identificando:

As utilizações do limiar local, usando, individualmente, soma e média, como limiar apresentaram os piores resultados obtidos durante os experimentos, não apresentando valores significativos, por isso sua analise foi pouco detalhada neste artigo;

O uso do limiar de Decisão associada à Distância Euclidiana apresentou resultados superiores aos demais. Entretanto, o mesmo não apresentou bons resultados quando utilizado um número de assinaturas de referência igual a seis, associado a um número fixo de pontos;

Quanto aos testes realizados foram levados em consideração cinco cenários, sendo, do primeiro ao quarto uma quantidade fixa de pontos, enquanto o quinto foi utilizado uma quantidade variável, de acordo com o autor. Observou-se que os melhores resultados para os experimentos com um número de pontos fixos foram obtidos para a quantidade de assinaturas de referência próximas a 12, mostrando que a uma convergência para o número de assinaturas próximo a 12 , e mesmo elevando o seu 
número os resultados não apresentam uma melhora significativa para as taxas de Erros, Global e Tipo II (falsa aceitação);

Verificou-se através dos experimentos que o melhor resultado foi obtido utilizando quantidade de pontos distintos entre os autores, chegando a se ter para o cenário I, resultado superior aos melhores obtidos com a utilização de pontos fixos.

Uma limitação da proposta foi utilização apenas da distância Euclidiana, como limiar de desição para validação da assinatura. Um trabalho futuro seria utilizar métricas que levem em consideração a deformação dos triângulos gerados para as imagens intermediarias.

\section{Referências}

ARMAND, S.; BLUMENSTEIN, M.; MUTHUKKUMARASAMY, V. Offline signature verification using the enhanced modified direction feature and neural-based classification. International Joint Conference on Neural Networks, 2006. 684-691.

BALTZAKIS, H.; PAPAMARKOS, N. A new signature verification technique based on a two stage neural network classifier. Engineering applications of Artificial intelligence, 14 Setembro 2000. 95-103.

BARANOSKI, F. L.; JUSTINO, E.; BORTOLOZZI, F. Identificação da Autoria em Documentos Manuscrito Usando SVM. XXV Congresso da Sociedade Brasileira de Computação. UNISINOS, 2005.

BATISTA, L. et al. State Of The Art In Off-line Signature Verification. Pattern Recognition technologies and Applications: Recent Advances, 2007. 39-53.

BERTOLINI, D. Agrupamento de Classificadores na Verificação de Assinaturas Offline. Dissertação de Mestrado, Pontifícia Universidade Católica do Paraná, Curitiba, 2008.

BERTOLINI, D.; OLIVEIRA, L. S.; JUSTINO, E. S. R. Reducing forgeries in writerindependent off-line signature verification through ensemble of classifiers. Pattern Recognition, v. 43, January 2010.

CARDOT, $\mathrm{H}$. et al. A static signature verification system based on a cooperating neural networks architecture. IJPRAI, v. 8, n. 3, p. 679-692, 1994.

COETZER, J. Off-line Signature Verification. PhD thesis, University of Stellenbosh, 2005.

DELAUNAY, B. Sur la sphère vide. Izvestia Akademii Nauk SSSR, Otdelenie Matematicheskikh i Estestvennykh Nauk, v. 7, p. 793-800, 1934.

FANG, B. et al. Off-line signature verification by the tracking of feature and stroke positions. Pattern Recognition, v. 36, n. 1, p. 91-101, 2003.

GONÇALVES, B. D. Agrupamento de Classificadores na Verificação de Assinaturas Off-line. Dissertação de Mestrado, Pontifícia Universidade Católica do Paraná, Curitiba, 2008.

GUEDES, A. L. P. Introdução à Geometria Computacional. www.inf.ufpr.br, 2011. Disponivel em: <http://www.inf.ufpr.br/andre/geom/geom.html>. Acesso em: 21 Agosto 2011. 
GUO, J. K.; DOERMANN, D. S.; ROSENFELD, A. Local correspondence for detecting random forgeries. In ICDAR 97, International Conference on Document Analysis and Recognition, Washington, DC, USA. IEEE Computer Society, 1997. 319-323.

HEINEN, M. R.; OSÓRIO, F. S. Autenticação de Assinaturas utilizando Análise de Componentes Principais e Redes Neurais Artificiais. SBIA-IBERAMIA 2006 / WCI - Simp. Brasileiro de Inteligência Artificial., Ribeirão Preto - SP, October 2006.

JUSTINO, E. J. R.; BORTOLOZZI, F.; SABOURIN, R. Offline signature verification using hmm for random. In ICDAR 2001, International Conference on Document Analysis and Recognition, 2001. 1031-1034.

JUSTINO, E. J. R.; BORTOLOZZI, F.; SABOURIN, R. A comparison of svm and hmm classifiers in the off-line signature verification. Pattern Recogn. Lett, v. 26, n. 9, p. 1377-1385, 2005.

KASS, M.; WITKIN, A.; TERZOPOULOS, D. Snakes: Active Contour Models. International Journal of Computer Vision, 1988. 321-331.

MA, ZENG. A Novel Off-line Signature Verification Based on Adaptive Multiresolution Wavelet Zero-Crossing and One-Class-One-Network. Lecture Notes in Computer Science, v. 4493/E, p. 4493-1077, 2007. ISSN 2007.

MIGHELL, D. A.; WILKINSON, T. S.; GOODMAN, J. W. Backpropagation and its application to handwritten signature verification. Advances in Neural Information Processing Systems 1, 1989. 340-347.

PLAMONDON, R.; LORETTE, G. Automatic signature verification and writer identification -- the state of the art. Pattern Recognition, v. 22, n. 2, p. 107-131, 1989.

SANTOS, C. R. Análise Automática de Assinaturas Manuscritas Baseada nos Princípios da Grafoscopia. Dissertação de Mestrado, Pontifícia Universidade Católica do Paraná, Curitiba, 2004.

SOUSA, M. Verificação de Assinaturas Off-line: Umaabordagem Baseada na Combinação de Distância e em Classificadores de uma Classe. Dissertação de Mestrado, Agosto 2009.

WEISSTEIN, E. W. Delaunay Triangulation. De MathWorld, 2011. Disponivel em: $<$ http://mathworld.wolfram.com/DelaunayTriangulation.html $>$. Acesso em: 11 Julho 2011.

YEUNG, D. Y. et al. First int. signature verification competition. 1st Int. Conf. Biometric Authentication (ICBA), Hong Kong, July 2004. 16-22.

SHUKLA, A. K.; MOHAN, P.; OJHA, G.; WARIYA, M. Issues and Challenges in Intelligent Computing Techniques (ICICT), 2014 International Conference on Digital Object Identifier: 10.1109/ICICICT.2014.6781380 Publication Year: 2014, Page(s): $784-789$. 